(C) 1996 IEEE. Personal use of this material is permitted. However, permission to reprint/republish this material

for advertising or promotional purposes or for creating new collective works for resale or redistribution to servers

or lists, or to reuse any copyrighted component of this work in other works must be obtained from the IEEE.

\title{
STUDY OF 14 GHZ VLEPP KLYSTRON WITH RF ABSORBING DRIFT TUBES
}

\author{
G.V. Dolbilov, N.I. Azorsky, A.A. Fateev, N.I. Lebedev, V.A. Petrov, V.P. Sarantsev, \\ V.S. Shvetsov, M.V. Yurkov \\ Joint Institute for Nuclear Research, 141980 Dubna, Moscow Region, Russia \\ V.E. Balakin, P.V. Avrakhov, V.I. Chashurin, S.Yu. Kazakov, N.A. Solyak, V.E. Teryaev \\ Branch of Budker Institute of Nuclear Physics, 142284 Protvino, Moscow Region, Russia
}

Results of experimental study of a wide-aperture relativistic klystron for VLEPP are presented. Investigations have been performed using the driving beam of the JINR LIA-3000 induction accelerator. To suppress self-excitation parasitic modes we have used technique of RF absorbing drift tubes. We have obtained $75 \mathrm{MW}$ of output power in a long pulse (250 ns) and $100 \mathrm{MW} \pm$ in a short pulse (50 $\mathrm{ns})$.

\section{Introduction}

To achieve a high output power of $X$-band klystron $(\sim 100 \mathrm{MW})$ at a moderate value of the emittance of the driving beam, the aperture of the klystron should be done as large as possible. On the other hand, at increasing the aperture, the frequencies of the parasitic modes become quite close to the operating frequency and their increments grow with the beam current which makes the problem of the parasitic oscillation suppression more complicated. Our experience have shown that standard techniques to suppress parasitic oscillations (the use of the wave chokes and the technique of permanent change of the phase velocity of the parasitic modes to decrease the interaction region of the beam with parasitic modes) do not provide the desired results, especially, in the case of a high gain $(\sim 70-80 \mathrm{~dB})[2]$, [3].

In papers [2], [3] we have proposed another idea to suppress parasitic oscillations which consists the use of RF absorbing drift tubes for distributed suppression of parasitic oscillations. We have upgraded the $11 \mathrm{~mm}$ aperture klystron with RF absorbing insertions and obtained encouraging results: the self-excitation modes have been suppressed and we have achieved the value of the output RF power about of $45 \mathrm{MW}$ at the beam current $I \sim$ 150 A which corresponds to the designed value of the klystron ef®ciency $30 \%$. A damage of output structure and wave transformer due to high RF power forced us to stop experiments with this klystron [3].

In this paper we present the results of ampli®cation experiments with wide-aperture $(15 \mathrm{~mm})$ VLEPP klystron upgraded with absorbing drift tubes.

\section{Experimental setup}

Parameters of the klystron are presented in Table 1. Peculiar feature of this klystron is that it has large aperture of drift tubes $(15 \mathrm{~mm})$. This helps to increase acceptance of the klystron. Nevertheless, there is one harmful consequence of a large aperture \pm the ground $\mathrm{H}_{11}$ waveguide mode is not cut-off one for this klystron. As there are always misalignments of the elements of the klystron, this results in self-excitation of the klystron on the ground $\mathrm{H}_{11}$ mode at $14 \mathrm{GHz}$.

Investigations have been performed at JINR using the driving beam of LIA-3000 induction accelerator (energy $1 \mathrm{MeV}$, beam current up to $300 \mathrm{~A}$, beam emittance $0.05 \pi \mathrm{cm} \cdot \mathrm{rad}$, pulse duration $250 \mathrm{~ns}$ ). We have the possibility to measure the beam current at the accelerator exit, entrance and exit of the klystron and the beam current losses inside the klystron. To obtain a more detailed information about the RF radiation, we have used beam collector in a form of circular waveguide [4].

To study the beam dynamics in the focusing system we have screened the beam from the electromagnetic structure of the klystron by a thin-wall Ti tube. Measurements have shown that there were no losses of the current in the buncher and the output structure. The value of the beam current in the collector was $300 \mathrm{~A}$.

Investigations of the self-excitation regime have shown that

Table I

Parameters of wide-aperture VLEPP klystron

\begin{tabular}{ll} 
General parameters & \\
Beam voltage & $1 \mathrm{MeV}$ \\
Beam current & $250 \mathrm{~A}$ \\
RF frequency & $14.0 \mathrm{GHz}$ \\
Power gain & $80 \mathrm{~dB}$ \\
RF peak output power & $100 \mathrm{MW}$ \\
Ef囚ciency & $40 \%$ \\
Focusing system & \\
Type of magnets & Permanent magnet \\
Max. Magnetic ${ }^{\circ} \mathrm{eld}$ & $4.5 \mathrm{kGs}$ \\
Period & $64 \mathrm{~mm}$ \\
Number of periods & 14.5 \\
Acceptance & $0.1 \pi \mathrm{cm} \cdot \mathrm{rad}$ \\
Buncher & \\
Drift tube diameter & $15 \mathrm{~mm}$ \\
Length of drift section & $52 \mathrm{~mm}$ \\
Number of drift sections & 10 \\
Length of cavity & $12 \mathrm{~mm}$ \\
Number of cavities & 11 \\
Mode of operation & $\pi$ \\
Output structure & \\
Mode of operation & $\pi / 2$ \\
Number of cells & 22 \\
Length & $110 \mathrm{~mm}$ \\
Aperture & $20 \mathrm{~mm}$ \\
\hline
\end{tabular}



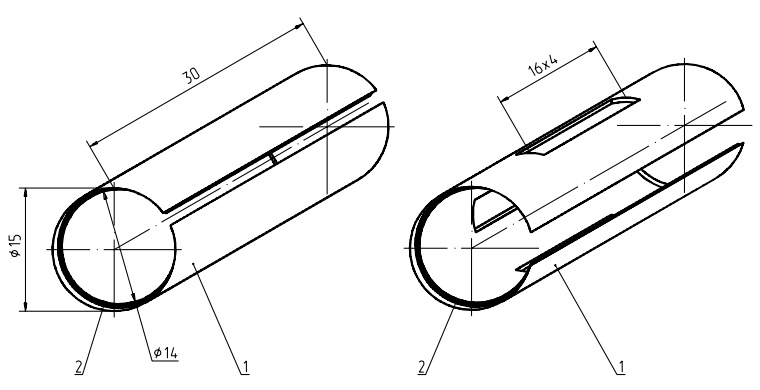

Figure. 1. Scheme of RF absorbing insertions. Here (1) \pm metal foil, (2) $\pm \mathrm{RF}$ absorbing layer. Insertion in the right-hand side is placed together with RF ®lter 6 (see Fig.2).

there exists strong self-excitation of parasitic oscillations in the klystron at frequency bands:

$$
\begin{aligned}
& 13.9 \mathrm{GHz} \lesssim f \lesssim 14.15 \mathrm{GHz}, \quad f_{1}=14.04 \mathrm{GHz} \\
& 16.3 \mathrm{GHz} \approx f \approx 16.50 \mathrm{GHz}, \quad f_{2}=16.40 \mathrm{GHz}
\end{aligned}
$$

and their harmonics [4]. Application of standard techniques to suppress the self-excitation was ineffective due to a high gain and a wide aperture of the klystron [4]. The presence of the signal from master oscillator (TWT) does not change the situation.

\section{Upgrading of the klystron with RF absorbing drift tubes}

In papers [2], [3], [4] we have developed an idea to suppress parasitic oscillations which consists the use of RF absorbing drift tubes for distributed suppression of parasitic oscillations. The main idea of this approach is to $®$ nd such a klystron design where the increments of parasitic modes are less than their attenuation in the klystron. We have realized this concept in the following way. We have developed technology of RF attenuating insertions and placed them inside the drift tubes of the klystron (see Figs.1 and 2). We have studied several methods to obtain absorbing materials. Investigations have shown that glass-carbon materials are more simple for manufacturing and use in our equipment.

Such a distributed suppression ${ }^{\circledR}$ lter provides signi $®$ cant attenuation of the parasitic modes and does not perturb the klystron operating mode [4]. Operating experience has shown that insertions do not affect vacuum conditions and are stable to the heat and radiation load.

We have expected also that the insertions may cause resistive instabilities of the beam. Nevertheless, thorough investigations of the beam dynamics have not shown any evidence of such instabilities.

In the same way as it has been described in the previous section, we have performed the study of the self-excitation mode of operation. It was found that all parasitic modes of the selfexcitation have been totally suppressed.

\section{Study of ampli®cation regime}

After upgrading of the klystron with RF absorbing insertions, we have performed the study of ampli®cation regime. The master signal was generated by the travelling wave tube. Typical

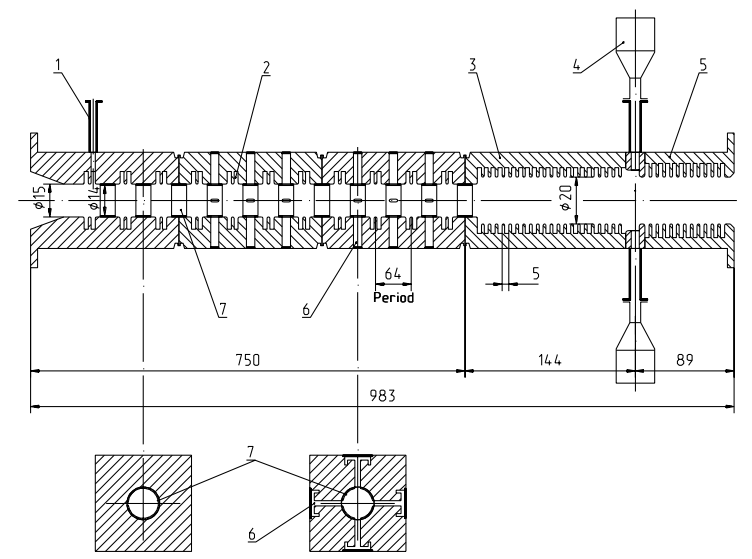

Figure. 2. Layout of the klystron with RF absorbing insertions. Here (1) \pm input waveguide, (2) \pm resonators of buncher, (3) \pm output structure, (4) \pm RF load, (5) \pm RF $® 1$ lter for $\mathrm{E}_{1}$ mode, (6) $\pm \mathrm{RF}$ (1ters for $\mathrm{H}_{1}$ modes $(14 \mathrm{GHz}$ and $16.4 \mathrm{GHz}),(7) \pm \mathrm{RF}$ absorbing insertions (placed inside drift tubes).

oscillograms of ampli®cation mode of operation are presented in Fig.3. It is seen from Fig.3a that there are no -uctuations of the beam current in the collector which indicates on the absence of the transverse beam instabilities. We have measured the frequency spectrum of the output radiation and have not observed any frequencies except of operating frequency $14 \mathrm{GHz}$.

At the beginning of operation at the level of output power of about $10 \mathrm{MW}$ we have obtained that there are temporal instabilities in the form of output signal. At further increasing of the output power we have obtained shortening of the RF pulse with respect to the beam current pulse. This is connected with the RF discharges in the output structure. During RF training procedure [3], [4] we have gradually increased the value of the output power and after $3 \times 10^{5}$ pulses we have reached $75 \mathrm{MW}$ output power within the pulse length of 250 ns. In Fig.4 we present amplitude characteristic of the klystron. It is seen that there is good agreement between theoretical and experimental results.

Upon achieving the level of output power of $75 \mathrm{MW}$ the ef®ciency of training diminished signi ${ }^{\circledR}$ cantly. short pulse $(50 \mathrm{~ns})$ we have achieved the value of the output RF power about of $100 \mathrm{MW}$ at the beam current $I \sim 290 \mathrm{~A}$.

\section{Conclusion}

In this paper we developed the concept of the klystron with distributed suppression of parasitic modes. Peculiar feature of the present experiment is that we proved experimentally a possibility to construct wide-aperture klystrons with aperture comparable with the RF wavelength. Such a klystron design possesses signi®cant advantages with respect to standard design revealing perspective of increasing operating current and, as a result, peak output power. We believe that wide-aperture klystrons with RF absorbing drift tubes can form a novel direction in the design of short RF wavelength klystrons.

\section{Acknowledgments}

We wish to thank A.M. Biryukov, V.I. Klementyev, V.V. Kosukhin, A.F. Kratko, N.A. Leonov and V.I. Skorlupkin for tech- 
(a)

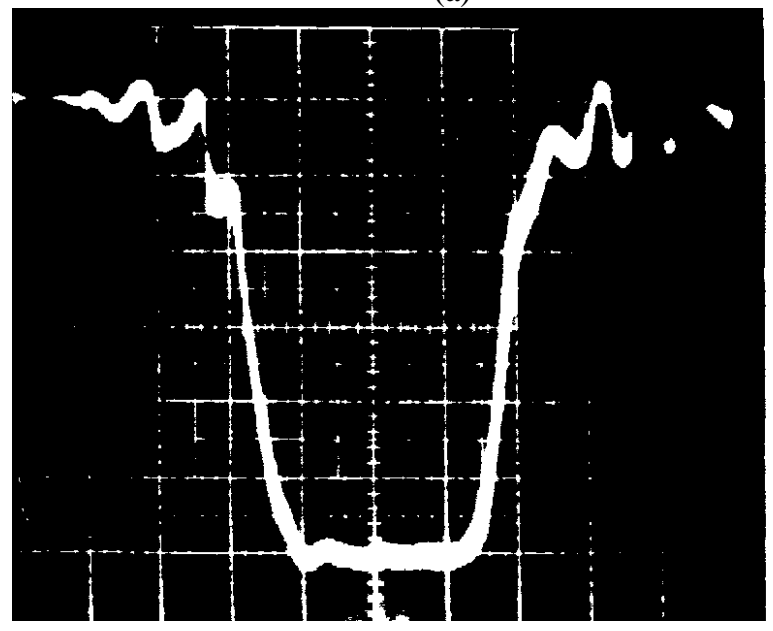

(b)

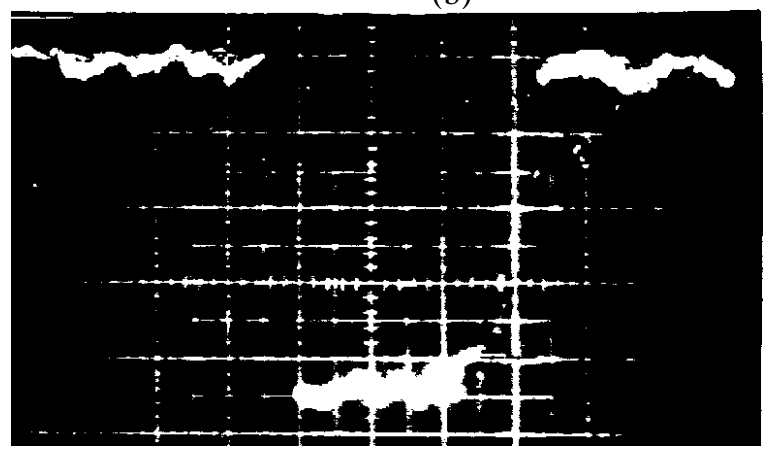

(c)

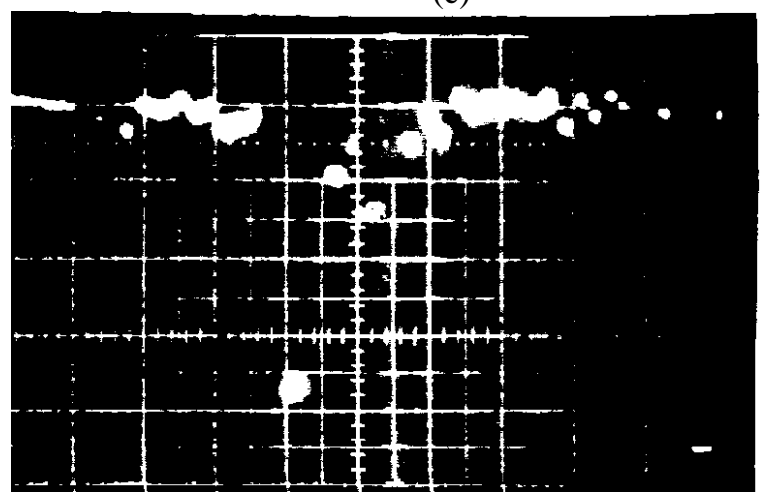

Figure. 3. Oscillogram of the ampli®cation regime. Here (a) the beam current in collector, (b) - RF signal after RF training cycle ( $\mathrm{P}=75 \mathrm{MW}),(\mathrm{c})$ - RF signal at the beginning of the next RF training cycle (maximum corresponds peak power of $100 \mathrm{MW}$

nical assistance in preparing and performing experiment.

\section{References}

[1] N.I. Azorskiy et al., ${ }^{\text {a VLEPP Klystron Activity at Dubna }}{ }^{\circ}$, Proc. of the International Workshop on Pulsed RF Power Sources for Linear Colliders (July 5-9, 1993, Dubna, Russia), p.143

[2] N.I. Azorsky et al., a Study of the $14 \mathrm{GHz}$ relativistic klystron for $\mathrm{VLEPP}^{\circ}$, presented at 17 th International Linac Conference (Tsukuba, Japan, 1994).

[3] G.V. Dolbilov et al., 'Study of $14 \mathrm{GHz}$ VLEPP Klystrons with 11 and $15 \mathrm{~mm}$ Aperture $^{\circ}$, presented at the Workshop

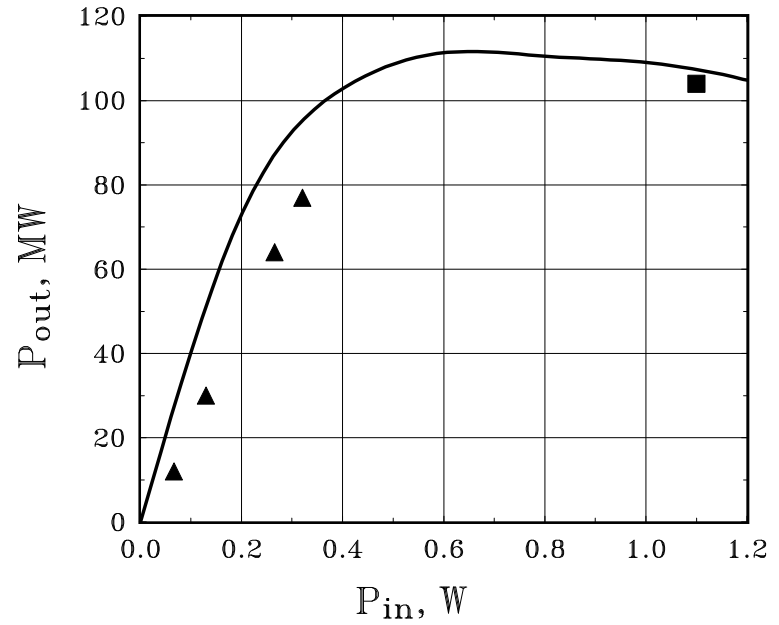

Figure. 4. Amplitude characteristic of the klystron. Here solid curve \pm theoretical calculations at $I=250 \mathrm{~A}, \boldsymbol{\Delta} \pm$ output power in a long pulse (250 ns) and \pm output power in a short pulse (50 ns).

on Pulsed RF Sources for Linear Colliders (Montauk, Long Island, New York, 1994)

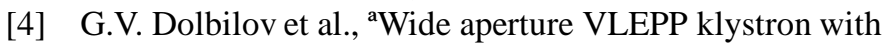
absorbing drift tubes ${ }^{\circ}$ presented at the 6 th International Workshop on Linear Colliders (Tsukuba, Japan, 1995) 